\title{
Focus on pelvis and acetabulum
}

\author{
Ramesh Kumar Sen ${ }^{1} \cdot$ Pol Maria Rommens ${ }^{2}$
}

Received: 5 March 2015 / Accepted: 11 March 2015 / Published online: 21 April 2015

(C) Springer-Verlag Berlin Heidelberg 2015

Fractures of the pelvic ring and acetabulum remain to be a major challenge. There are diverse reasons for this. Pelvic and acetabular lesions are relatively seldom, and there is a long learning curve for the orthopedic trauma surgeon. The pelvic ring, especially the innominate bone, is a complex three-dimensional structure. Analysis of injuries of this large osteoligamentar construct is demanding. Classification of pelvic and acetabular lesions is also difficult; it requires a thorough assessment of conventional radiographs and CT images with multiplanar reconstructions. Finally, surgery of the pelvis and acetabulum is dangerous, as vital neurovascular structures pass near to the fractures.

It therefore is understandable yet problematic that surgeons delay the decision to operate or not on these lesions. This has several unfavorable consequences. The outcome is worse when pelvic fractures are operated on later than 3 weeks after the accident. In vertically unstable pelvic ring lesions, it becomes very difficult to correct the vertical displacement. Also in acetabular injuries, precise reduction in displaced fracture fragments is hard when performed late. Letournel impressively demonstrated in his very large series that delayed surgery of acetabular fractures is connected with more complications and poor long-term outcome.

This "focus on" issue deals with late surgery and revision surgery of pelvis and acetabular injuries. Tripathy

R. K. Sen is a Former Professor in PGIMER, Chandigarh.

Pol Maria Rommens

pol.rommens@unimedizin-mainz.de

Department of Orthopaedics, Fortis Hospital, Mohali, India

2 Department of Orthopaedics and Traumatology, University Medical Center, Johannes Gutenberg-University, Langenbeckstrasse 1, Mainz 55131, Germany et al. [1] discuss the specific characteristics of pelvic nonunion and malunion. They collected the data of ten series between 2 and 204 patients. Pain, leg length discrepancy and instability are the predominant symptoms. They present a three-stage procedure for surgical correction, possible complications and results. Although outcome is unpredictable, operative treatment is mostly the only way to improve function.

Veerappa et al. [2] zoom in on neglected acetabular fractures. In order to decide on the optimal treatment, a thorough preoperative analysis of the situation is necessary. Hip dislocation, protrusion, cavitary bone defect and nonunion may be present. Open reduction and internal fixation may not be the ideal solution due to the severe damage on the femoral head. Treatment may differ from conservative to total hip arthroplasty. To secure solid cup implantation, bone grafting and anti-protrusio cup implantation may be necessary. Outcome will be considerably worse than after conventional hip replacement.

Two articles concentrate on pelvic fractures in the elderly. In their comprehensive article, Wagner et al. [3] review the characteristics of fragility fractures of the sacrum and present the typical distribution of bone density in the sacrum of elderly persons. As a consequence, the morphology of sacral fractures in the elderly is consistent, but stability varies from stable to highly unstable. When there is suspicion of a sacral lesion, conventional radiographs must be completed with $\mathrm{CT}$ imaging and MRI. Wagner et al. give an overview of possible treatment alternatives and discuss their advantages and limitations.

Dietz et al. [4] present a small but alarming series of fragility fractures of the pelvis with hemodynamic instability. Although seldom, arterial bleeding after pubic rami fractures may occur and there must be a high index of suspicion in the elderly patient, who suffered a pubic rami 
fracture after a fall. Inpatient observation for at least 1 day is recommended.

This "focus on" is an appeal for early and complete diagnosis of pelvis and acetabular injuries, thorough analysis and early decision making. We wish our readers will have a better understanding of the problems of delayed surgery. Sacral insufficiency fractures and active bleeding after fragility fractures of the pelvis are other challenges, with which we will be confronted increasingly in the future.

Conflict of interest Ramesh Kumar Sen and Pol Maria Rommens declare that they have no conflict of interest.

Compliance with ethical requirements This work is in compliance with ethical requirements. Ramesh Kumar Sen and Pol Maria Rommens declare that this is an editorial that includes no studies on humans or animals.

\section{References}

1. Tripathy SK, Goyal T, Sen RK (2014) Nonunions and malunions of the pelvis. Eur J Trauma Emerg Surg. 2015. doi:10.1007/ s00068-014-0461-0. (Published online November 4th 2014).

2. Veerappa LA, Tripathy SK, Sen RK. Management of neglected acetabular fractures. Eur J Trauma Emerg Surg. 2015. doi:10.1007/s00068-014-0462-z. (Published online November 4th 2014).

3. Wagner D, Ossendorf C, Gruszka D, Hofmann A, Rommens PM. Fragility fractures of the sacrum. How to identify and when to treat surgically? Eur J Trauma Emerg Surg. 2015. doi:10.1007/ s00068-015-0530-z.

4. Dietz SO, Hofmann A, Rommens PM. Hemorrhage in fragility fractures of the pelvis. 2015. doi:10.1007/s00068-014-0452-1. (Published online September 23rd 2014). 